Revista Complutense de Educación

ISSNe: 1988-2793

http://dx.doi.org/10.5209/RCED.57163

\title{
Fundamentos de la investigación y la innovación educativa
}

Autores: Enrique Navarro (Coordinador), Eva Jiménez, Soledad Rappoport y Bianca Thoilliez Editorial: Unir editorial

Año de publicación: 2017

$N^{a}$ de páginas: 276

ISBN: 978-84-16602-55-1

La finalidad de esta obra es ofrecer un manual básico que permita diseñar y poner en marcha proyectos de investigación e innovación educativa. Los autores recogen las bases de la metodología de la investigación educativa tratando la información de manera sistemática, además de aplicarla y acercarla al aula a través de la innovación educativa. Se realiza un recorrido por los diferentes diseños, así como las numerosas herramientas que los profesionales de la educación pueden utilizar en el aula, despertando la curiosidad del lector para responder a la explicación de fenómenos educativos comunes, al estudio de un caso en profundidad y/o a la transformación de la realidad educativa desde la acción práctica.

La obra se divide en nueve capítulos: los tres primeros capítulos, marcan las bases de la investigación e innovación educativa haciendo referencia a la relación entre ambas. Se detallan las fases del proceso y los elementos de un proyecto de investigación e innovación, así como el proceso de toma de decisiones y su redacción. De mismo modo, estos capítulos constan de diferentes epígrafes de gran utilidad para conectar el trabajo investigador con la realidad educativa, como por ejemplo: la funcionalidad de la investigación y la innovación, la ética investigadora, posibles áreas de investigación, un listado de bases de datos de búsqueda de información y de gestores bibliográficos con sus diferentes características y una serie de errores comunes que los profesionales en formación en investigación e innovación deben conocer. Los capítulos cuatro, cinco y seis se aúnan para una misma cuestión ¿Cómo puedo actuar? Para ello, estos capítulos comprenden los diferentes diseños que empleamos para resolver un problema de investigación, ya sea de corte cuantitativo (diseños experimentales, cuasi experimentales, pre-experimentales, no experimentales o expostfacto), cualitativo (la etnografía, estudio de caso, método biográfico-narrativo, teoría fundamentada y la investigación-acción) o aplicado (aprendizaje basado en problemas, gamificación, flipped classroom). El capítulo siete describe el proceso de recogida de información, además de las técnicas y herramientas más usuales que se emplean para tal fin. Este manual finaliza con dos capítulos centrados en destacar los procedimientos de análisis de información necesarios que permiten tener certeza de la consecución de los objetivos de los proyectos de investigación e innovación, en otras palabras, la ruta para la extracción de los resultados tanto en diseños cuantitativos como cualitativos.

A nivel global, la obra destaca por establecer la relación de la investigación y la innovación educativa poniendo en común que ambas parten de un estudio de diagnóstico y necesidades, así como de una fase primordial en ambos procesos que es la evaluación. A partir de estas fases primero se proponen los objetivos y, poste- 
riormente, gracias a la evaluación, se permite conocer si han sido alcanzados. Un ejemplo de nexo entre ambos procesos es el diseño de investigación - acción en el aula que engloba lo que sería la relación de la investigación e innovación. No obstante, todo proceso depende en primer lugar de la formulación de la pregunta de investigación y los objetivos planteados que facilita el empleo de una determinada metodología, herramientas y técnicas. Los autores señalan la importancia de recabar información previa que nos faciliten aquellas investigaciones que ya se hayan realizado y saber aquello que está todavía por hacer con el propósito de entender qué ocurre en determinadas situaciones. Por ello, la obra está conectada con la realidad de cada profesional educativo y del momento, en el que se encuentra, ya sea en los diferentes cursos como estudiante, en un centro educativo, asociación... lo primordial es cuestionarse un problema real y dar respuesta con conocimiento de las diferentes posibilidades que la investigación y la innovación educativa te ofrece.

De igual modo, las herramientas se utilizan en función de nuestros objetivos, actuando como un medio pero no como un fin para hacer investigaciones o innovaciones concretas. Por esta razón los autores subrayan el papel que juegan las TIC dentro del contexto educativo y su impacto para innovar y mejorar los procesos de enseñanza y aprendizaje, siempre y cuando se realice un uso correcto y didáctico de las mismas.

Otro de los puntos clave que subyace de la relación entre investigación e innovación educativa en la obra son los elementos del proyecto de investigación-innovación exponiendo la diferenciación entre la estructura de la investigación básica y de la investigación aplicada. En dichas estructuras podemos ver los apartados comunes de ambos proyectos, aunque en la parte de aplicación o plan de actuación es donde podemos ver mayor diferenciación, por ejemplo: la contextualización del proyecto no suele estar en la investigación básica, también en la investigación aplicada es clave describir de manera concreta los pasos que debe seguir un proyecto con la particularidad de que una persona externa al mismo sea capaz de aplicarlo sin dificultades.

En resumen, este manual se caracteriza por hacer "fácil lo difícil", proporcionando los conocimientos necesarios para el desarrollo de la reflexión educativa mediante la investigación e innovación educativa. Además, en cada capítulo se plantea una serie de actividades acorde a cada uno de sus objetivos con la finalidad de facilitar la adquisición del conocimiento y despertad el espíritu investigador de los profesionales educativos que tienen en sus manos el cambio educativo.

Víctor León Carrascosa victorleoncarrascosa@ucm.es Universidad Complutense de Madrid 